\title{
Novel Neuromuscular Electrical Stimulation System for Treatment of Dysphagia after Brain Injury
}

\author{
Keiichi Toyama, ${ }^{1}$ Shuji Matsumoto, ${ }^{2}$ Miho Kurasawa, ${ }^{1}$ Haruka Setoguchi, ${ }^{1}$ \\ Tomokazu NomA, ${ }^{1}$ Keita TaKenAKA, ${ }^{3}$ Akina SoEdA, ${ }^{3}$ Megumi SHIMOdOzOnO, ${ }^{2}$ \\ and Kazumi KAWAHIRA ${ }^{2}$ \\ ${ }^{1}$ Department of Rehabilitation, Kirishima Rehabilitation Center of \\ Kagoshima University Hospital, Kirishima, Kagoshima; \\ ${ }^{2}$ Department of Rehabilitation and Physical Medicine, Graduate School of Medical and \\ Dental Sciences, Kagoshima University, Kagoshima, Kagoshima; \\ ${ }^{3}$ Department of Rehabilitation, Tarumizu Municipal Medical Center, \\ Tarumizu Chuo Hospital, Tarumizu, Kagoshima
}

\begin{abstract}
The purpose of this study was to compare the effects of a novel neuromuscular electrical stimulation (NMES) to the effects of conventional treatment in patients with dysphagia after brain injury. In total, 26 patients were non-randomly divided into an experimental group $(n=12)$ and a control group $(n=14)$. The experimental group received NMES intervention followed by conventional treatment, including thermaltactile stimulation with intensive repetition of a dry-swallow task. The control group received conventional treatment without NMES. NMES at a fixed pulse duration of 50 us and a frequency of $50 \mathrm{~Hz}$ was delivered over the skin areas above the motor point of the target muscles, i.e., the bilateral geniohyoid, mylohyoid/anterior belly of the digastric, and thyrohyoid muscles, using a high-voltage pulsed-current device. The two groups received 40-min treatments once a day, 5 days per week, for 8 weeks. Outcome, assessed before and 8 weeks after treatment, was evaluated with regard to the videofluoroscopic dysphagia scale (VDS), the anterior and superior displacement of the hyoid bone and larynx, and the functional oral intake scale. Both groups exhibited improvement, but the experimental group exhibited more significant improvement in the displacement of the hyoid bone and larynx, VDS-total score, and VDS-pharyngeal score than the control group did. The results suggest that NMES combined with conventional treatment is superior to conventional treatment alone in patients with dysphagia following treatment for brain injury. Further investigations are necessary to examine the effects of NMES in patients with more varied types of diseases.
\end{abstract}

Key words: dysphagia, neuromuscular electrical stimulation, high-voltage pulsed current, hyoid, larynx

\section{Introduction}

Neuromuscular electrical stimulation (NMES) has been reported in several recent studies as a treatment for pharyngeal dysphagia. ${ }^{1-5)}$ The primary principle underlying this approach is that electrical stimulation can enhance muscle function during swallowing activity. ${ }^{6,7)}$ NMES uses surface electrodes to deliver electrical stimulation to muscles, causing muscle contractions by depolarization of nerve fibers within the region of application..$^{8-12)}$

The use of electrical stimulation of the anterior neck muscles in treating dysphagia was first

Received October 31, 2013; Accepted December 16, 2013 reported by Freed et al. ${ }^{3)}$ In that study, they compared the effectiveness of NMES therapy with that of thermal-tactile stimulation (TTS) therapy. Patients who underwent each type of therapy made improvements in swallowing function, but those in the NMES group reported greater and longer-lasting benefits. Following publication of those findings, the NMES technique has been widely used as a treatment option. Surface electrodes are noninvasive, easily applied, and provide good recruitment of superficial muscles; however, stimulation of a specific muscle or of deep muscles is difficult. ${ }^{11,13)}$ In the reports of Humbert et al. ${ }^{14)}$ and Ludlow et al., ${ }^{15)}$ selective stimulation of the thyrohyoid muscle by surface electrodes was impossible. On the other 
hand, although implanted electrodes can selectively stimulate target muscles, they are invasive and expensive, and injection of the electrodes into the muscles requires expertise.

Leelamanit et al. reported their experience with synchronized electrical stimulation using highvoltage pulsed current (HVPC) in 23 persons with dysphagia, and concluded that dysphagia was improved in these patients. ${ }^{16)}$ HVPC is characterized by a twin-peak pulse monophasic waveform, generating high voltage and a low total current, with very short pulse duration and relatively larger interpulse intervals. These characteristics allow HVPC to generate little or no electrochemical reaction between the skin and the stimulation electrodes; consequently, the resultant sensations are comfortable for the patient. ${ }^{17,18)}$ Many reports support the use of HVPC for prevention and improvement of edema, acceleration of wound healing, reduction of pain, improvement of blood circulation, and muscle strengthening. ${ }^{18-21)}$ If a novel technique for NMES using HVPC could produce anterior-superior displacement of the hyoid bone and the larynx, it would be a valuable method for improving swallowing function in patients with dysphagia.

We previously developed a novel method of NMES using HVPC and an 8-mm diameter metal sphere monopolar stimulating electrode to accelerate the anterior-superior displacement of the hyoid bone and the larynx in healthy volunteers. Our pilot data suggested that the NMES appeared to be safe and feasible, and could selectively stimulate the suprahyoid and the thyrohyoid muscles in healthy volunteers, ${ }^{22)}$ suggesting that this method might provide an alternative treatment mechanism for dysphagia; however, those results were based on healthy people rather than patients with dysphagia.

In this study, we investigated the effects of the novel NMES system in dysphagic patients with brain injury, and compared them to the effects of conventional treatment.

\section{Materials and Methods}

\section{Subjects}

In total, 26 patients (22 male and 4 female; mean age \pm SD, $65.5 \pm 17.3$ years) were non-randomly divided into an experimental group $(\mathrm{n}=12)$ and a control group $(\mathrm{n}=14)$. The patients were recruited from among inpatients admitted to the Kirishima Rehabilitation Center of Kagoshima University Hospital or the Tarumizu Municipal Medical Center of Tarumizu Chuo Hospital, Japan, between 02 November 2009 and 30 July 2012.

The inclusion criteria were as follows: (1) diagnosis of dysphagia with brain injury; (2) biomechanical evidence of pharyngeal dysphagia per clinician judgment from a videofluoroscopic swallowing study (VFSS) (biomechanical evidence of pharyngeal dysphagia was defined as the presence of reduced displacement of the hyoid bone and larynx and reduced pharyngeal constriction); (3) brain injury without neurologic signs typical of brainstem involvement; and (4) a physician referral stating that the patient was in stable medical condition.

The exclusion criteria were as follows: (1) epilepsy or implanted cardiac pacemaker; (2) tumors or neoplastic disease of the swallowing apparatus and who had undergone radiotherapy to the neck; (3) previous surgery on the swallowing apparatus; (4) severe carotid stenosis and flexible plaque as revealed by carotid artery ultrasound; and (5) inability to give informed consent because of cognitive impairment or receptive aphasia. The general characteristics of the patients are given in Table 1.

Informed consent was obtained from each patient, once they fully understood the purpose and methodology, according to the ethical guidelines of the hospital. The study was approved by the Ethical Committee of Kagoshima University.

\section{Experimental procedure}

This study had a prospective observer-blinded open-label controlled design. The experimental group received NMES intervention followed by conventional treatment (including tongue exercise, TTS with intensive repetition of dry swallow, Mendelsohn maneuver, etc.). The control group received the same conventional treatment as the experimental group, without NMES. The two groups received 40 -min treatment once a day, 5 days per week, for 8 weeks. Treatment sessions followed a protocol as an 8-week program of lingual strength

Table 1 Characteristics of subjects

\begin{tabular}{lcc}
\hline Characteristics & $\begin{array}{c}\text { Experimental group } \\
(\mathrm{n}=12)\end{array}$ & $\begin{array}{c}\text { Control group } \\
(\mathrm{n}=14)\end{array}$ \\
\hline $\begin{array}{l}\text { Age, years, mean } \pm \\
\text { SD (range) }\end{array}$ & $\begin{array}{c}63.6 \pm 21.4 \\
(17-85)\end{array}$ & $\begin{array}{c}67.2 \pm 13.7 \\
(33-83)\end{array}$ \\
$\begin{array}{l}\text { Gender, male/ } \\
\text { female, } \mathrm{n}\end{array}$ & $12 / 0$ & $10 / 4$ \\
$\begin{array}{l}\text { Infarction/ } \\
\text { Hemorrhage/ } \\
\text { others, } \mathrm{n}\end{array}$ & $7 / 5 / 1$ & $7 / 4 / 3$ \\
$\begin{array}{l}\text { Time since onset } \\
\text { of stroke, weeks, } \\
\text { mean } \pm \text { SD (range) }\end{array}$ & $25.2 \pm 25.9$ & $14.7 \pm 10.6$ \\
& $(8-106)$ & $(7-49)$ \\
\hline
\end{tabular}

SD: standard deviation. 
exercises for dysphagia. ${ }^{23)}$

\section{Interventions}

NMES system: Electrical stimulation was delivered using an HVPC device (Universal Stimulation Current Unit ES-530, ITO Co., Ltd., Tokyo) (Fig. 1A). The waveform produced by the stimulator was twinpeak monophasic pulsed current. As mentioned above, HVPC is characterized by a twin-peak pulse monophasic waveform, generating a high voltage and a low total current, with very short duration and longer inter-pulse intervals (Fig. 2). ${ }^{17,18)}$

Two sets of electrodes were used in this study. Active electrodes (negative polarity) were applied to a metal-sphere probe (composite of 8-mm diameter metal sphere and on/off switch) (Fig. 1B). Dispersive electrodes were applied to a gel pad (square, $100 \times 50 \mathrm{~mm}$ ). With the switch pressed, the current was delivered to the target muscles. Monopolar stimulation was used in this NMES system. The target muscles were the geniohyoid, the mylohyoid/anterior belly of the digastric, and thyrohyoid muscles. Stimulation region was identified as the skin area above the anatomically defined motor points of the geniohyoid $2.1 \mathrm{~cm}$ superior from the superior end of the hyoid bone and $0.8 \mathrm{~cm}$ lateral from the midline), the mylohyoid/anterior belly of the digastric $(2.3 \mathrm{~cm}$ superior from the superior end of the hyoid bone and $2.2 \mathrm{~cm}$ lateral from the midline), and thyrohyoid muscles (lateral and inferior of the superior thyroid tubercle), as described in a previous report. ${ }^{13)}$ The active electrodes were delivered bilaterally over the motor points of the target muscles, and the dispersive electrodes were placed bilaterally over the cervical spine (Fig. 3). The pulse duration and the frequency were fixed at $50 \mathrm{msec}$ and $50 \mathrm{~Hz}$, respectively. The intensity was adjusted to a level of muscle contraction that was not uncomfortable. These settings were selected based on the results
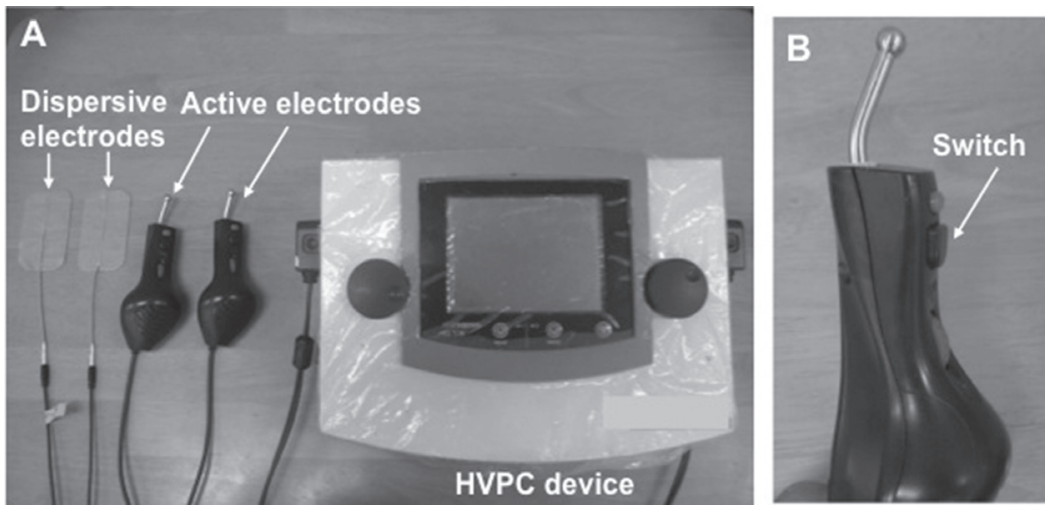

Fig. 1 Electrical stimulation was delivered using an high-voltage pulsed current (HVPC) device with a direct monophasic pulsed current and twin-peak pulse. A: HVPC device composite of active electrodes (8-mm diameter metal sphere electrode) and dispersive electrodes (gel pad), B: Active electrode.

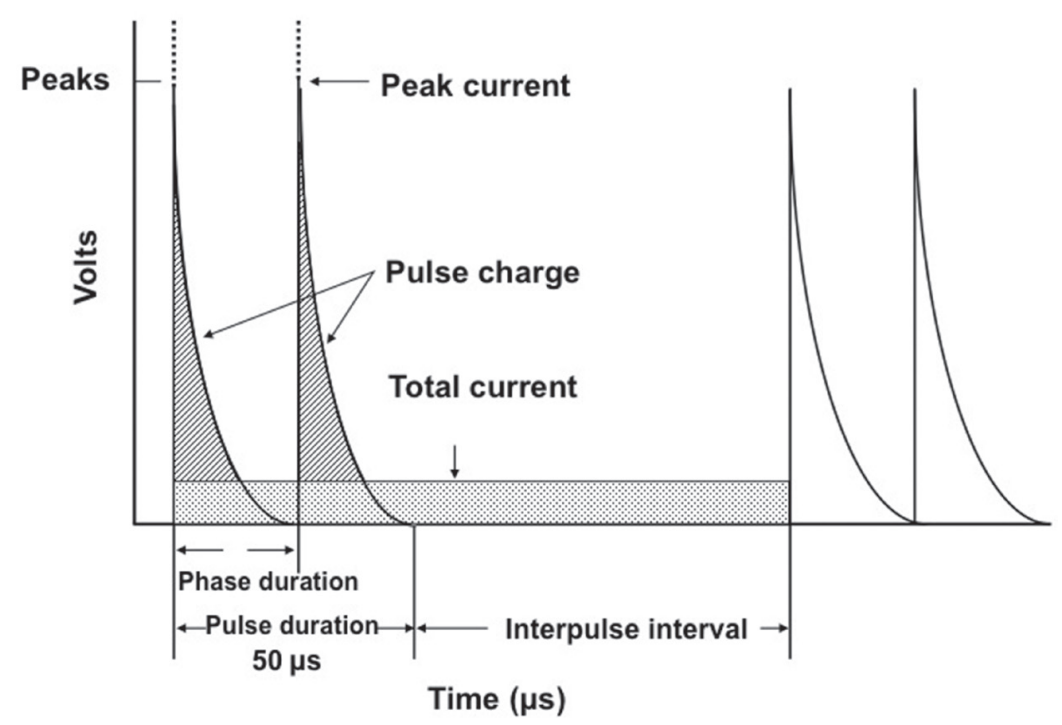

Fig. 2 Schematic diagram of high-voltage pulsed current (HVPC). Output waveforms from Universal Stimulation Current Unit ES-530 showing twin-peak pulse monophasic waveform, generating high voltage and a low total current, with very short duration and longer inter-pulse intervals. 
of previous studies. ${ }^{22}$

NMES intervention: Before NMES intervention, we confirmed the displacement of the hyoid bone and larynx during electrical stimulation in all subjects using VFSS, and identified motor points according to the stimulation regions, as described above.

The NMES intervention was conducted by a speech-language-hearing therapist. The patient was seated in a wheelchair in an upright position. We had previously put marks on the neck that indicated where the motor points were identified. The clinician stimulated the motor-point marks and controlled the onset of electrical stimulation by pressing a switch. To each session, following NMES treatment, we added TTS with intensive repetition of a dryswallow task. One session consisted of three 10-min sets (geniohyoid muscle, mylohyoid/anterior belly of the digastric muscle, thyrohyoid muscle) separated by 2 min of rest to prevent fatigue.

\section{Assessment}

Outcome was assessed before and 8 weeks after treatment. We evaluated the videofluoroscopic dysphagia scale (VDS), ${ }^{24)}$ the displacement of anterior and superior hyoid bone and larynx, and the functional oral intake scale (FOIS). ${ }^{25)}$ To confirm feasibility and safety, blood pressure, heart rate, and percutaneous $\mathrm{O}_{2}$ saturation were also assessed before, during, and after each trial.

VDS: Both groups received VFSS before intervention and at the end of treatment in order to measure functional changes in swallowing. The fluoroscopic tube was focused in a lateral plane on the oral cavity from the lips anteriorly to the pharyngeal wall posteriorly, and from the nasopharynx superiorly to just below the upper esophageal sphincter area. Each patient was given $1 \mathrm{~cm} \times 1 \mathrm{~cm}$ and $1.5 \mathrm{~cm} \times 1.5 \mathrm{~cm}$ test boluses. The capture rate was 30 frames/s.

The VDS score (range, 0-100; superior score $=0$; inferior score $=100$ ) was developed to comprehensively reflect the swallowing functions of stroke patients based on VFSS findings. The 14 items in VDS represent oral (lip closure, bolus formation, mastication, apraxia, premature bolus loss, and oral transit time) and pharyngeal (pharyngeal triggering, vallecular and pyriform sinus residues, laryngeal elevation and epiglottic closure, pharyngeal coating, pharyngeal transit time, and aspiration) function. The maximum score for pharyngeal function (VDSpharyngeal) is 60 (superior score $=0$, inferior score $=$ 60 ), and the maximum score for oral function (VDSoral) is 40 (superior score $=0$, inferior score $=40$ ). Hyoid bone and laryngeal movement analysis: We used an Image Tracker PTV ver. 7.2 ${ }^{\circledR}$ (Digimo Co., Ltd., Osaka) for analysis of anterior-superior hyoid bone movement and laryngeal elevation. The investigator used a cursor to identify the points on the most anterior-inferior corners of the second and fourth vertebrae on each video frame, and a straight line was drawn between these two points to define the y-axis. When either the second or the fourth vertebra was not visible, the bottom anterior-inferior corners of the first and third vertebrae were used in the same fashion. A line perpendicular to the $\mathrm{y}$-axis at the anterior-inferior corner of the lower vertebra served as the x-axis. The following points were marked on each frame: the superior/inferior aspect of the subglottal air column measured the laryngeal position on the y-axis, and the anterior/posterior and the most superior/inferior point of the hyoid bone measured the position on the $\mathrm{x}$ - and $\mathrm{y}$-axis. ${ }^{14)}$ The displacement of the hyoid bone and laryngeal elevation was defined as the distance

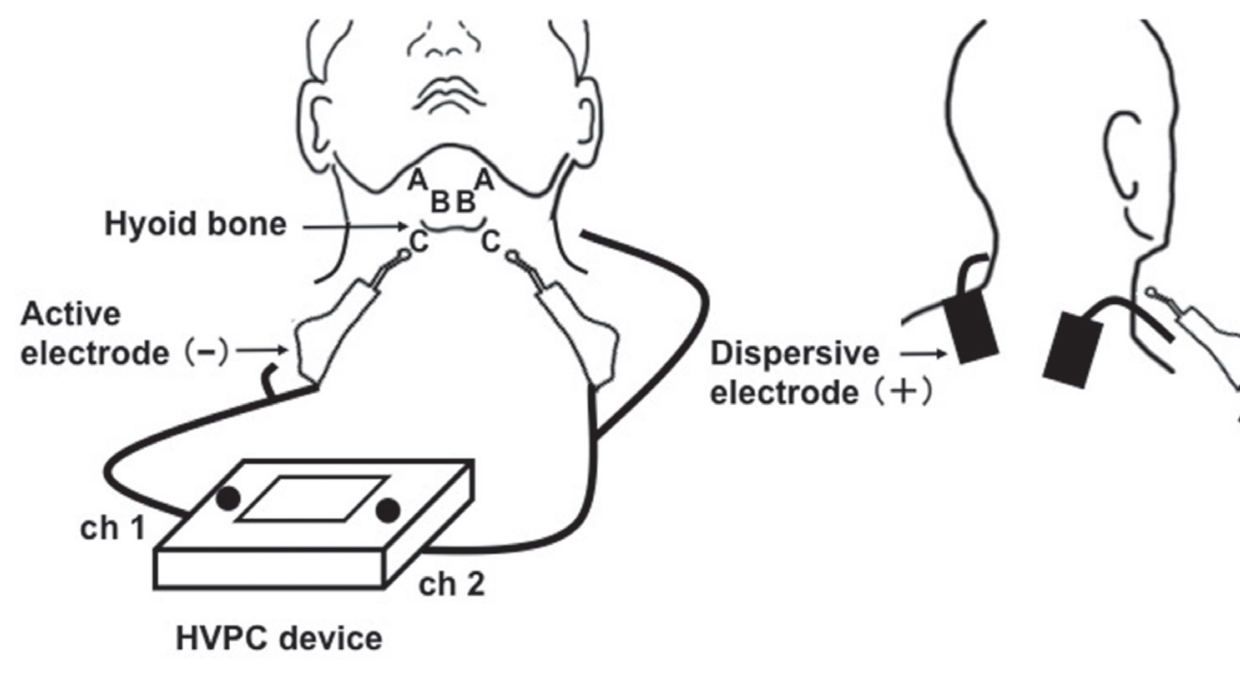

Fig. 3 Schematic of the stimulation region of the surface electrodes. The active electrodes were delivered bilaterally over the motor points of the target muscles, and the dispersive electrodes were placed over the cervical spine. A: mylohyoid/anterior belly of digastric muscles, B: geniohyoid muscles, C: thyrohyoid muscles. 
from the resting position to the highest position during swallowing trials. Distance measurements were made after calibration of the digitized image using a 2.0-cm diameter wire loop taped to the chin of the patient; data were transformed into actual distances using the dimensions of the wire.

FOIS: The FOIS is strictly based on the patient's ability to orally consume various consistencies of food and liquid, and it reveals specifics about the food textures and liquid consistencies that the patient can tolerate. This dietary scale consists of seven levels that range from Level 1 (a patient who is unable to orally consume any food or liquid) to Level 7 (a patient who is consuming an oral diet with no restrictions).

\section{Statistical analysis}

Data are presented as means \pm standard deviation (SD) of the mean. The VDS score and the FOIS score were analyzed using Wilcoxon signed-rank tests comparing the baseline values with the values at the completion of treatment. The displacement of the anterior and superior hyoid bone and larynx were analyzed using paired $t$-test of baseline values compared with the values at the completion of treatment. Differences in effect data between the experimental group and the control group were tested for significance using the Mann-Whitney U test. All statistical analyses were performed using IBM SPSS Statistics 18.0 (SPSS Inc., Chicago, Illinois, USA). Differences were considered significant when the $\mathrm{p}$ value was $<0.05$.

\section{Results}

None of the patients experienced discomfort before, during, or after the intervention. Electrical stimulation could be applied safely, and NMES produced neither adverse effects nor complications such as hypotension, arrhythmia, or syncope resulting from carotid body stimulation and/or laryngeal spasm.

All data from this study are summarized in Table 2. Significant improvements were observed in all parameters in the experimental group. By contrast, the control group exhibited no significant improvement in hyoid bone and laryngeal elevation after treatment.

The VDS-total and VDS-pharyngeal scores in the experimental group were statistically significantly different from those of the control group $(p<0.01)$ (Fig. 4A).

The average change in anterior displacement of the hyoid bone was significantly greater in the experimental group $(3.4 \pm 2.5$ versus $0.4 \pm 2.5$, $\mathrm{p}<0.01)$. The average change in the superior displacement of the hyoid bone was significantly greater in the experimental group $(7.9 \pm 6.5 \mathrm{~mm}$ versus $1.0 \pm 3.8 \mathrm{~mm}, \mathrm{p}<0.01)$. The average change in the superior displacement of the larynx was significantly greater in the experimental group $(9.1 \pm 5.0 \mathrm{~mm}$ versus $1.9 \pm 4.0 \mathrm{~mm}, \mathrm{p}<0.01)$. Comparison of the changes in the anterior displacement of the hyoid bone, the superior displacement of the hyoid bone, and laryngeal elevation between the two groups revealed a statistically significant improvement (Fig. 4B).

The mean FOIS values changed significantly, from 3.8 to 5.2 in the experimental group $(\mathrm{p}<0.05)$ and from 4.0 to 4.6 in the control group ( $p<0.05$ ). There was no difference between the two groups in the FOIS assessment.

\section{Discussion}

In this study, we showed that NMES to the geniohyoid, mylohyoid/ anterior belly of the digastric, and thyrohyoid muscles can be safely, feasibly, and effectively introduced into a novel treatment for dysphagic patients. Our results showed that the VDS score, the displacement of the hyoid bone and larynx, and swallowing function were significantly improved in patients who received NMES. To our knowledge, this study is the first report that NMES using HVPC and a localized metal sphere monopolar stimulating electrodes accelerate the extent of hyoid bone and laryngeal elevation and improve the swallowing function in dysphagic patients with brain injury.

Several studies have previously reported the effects of electrical stimulation on dysphagia. In patients with dysphagia caused by stroke, Lim et al. reported that NMES with surface electrodes placed on the submental region, between the thyroid cartilage and cricoid cartilage, and below the cricoid cartilage resulted in significant improvements in the swallow function scoring system, penetration-aspiration scale, and pharyngeal transit time compared with TTS alone. ${ }^{26)}$ Based on these findings, they concluded that NMES can enhance tone and strength or aid in muscle activation. Shaw et al. demonstrated that NMES with four different surface electrode configurations placed on the anterior neck muscles significantly improved dysphagia severity score, diet intake, penetration and aspiration, and residue severity in patients with dysphagia caused by stroke. ${ }^{27)}$ Furthermore, they showed that NMES increased laryngeal elevation in mild dysphagia. On the other hand, Bülow et al. studied the effects of NMES with surface electrodes placed on either side of the midline of the throat and above the level of the thyroid notch over the thyrohyoid muscle; that study 
Table 2 Changes in parameters before and after treatment

\begin{tabular}{|c|c|c|c|c|}
\hline & \multicolumn{2}{|c|}{ Experimental group } & \multicolumn{2}{|c|}{ Control group } \\
\hline & $\begin{array}{c}\text { Before } \\
\text { treatment }\end{array}$ & $\begin{array}{c}\text { After 8-week } \\
\text { treatment }\end{array}$ & $\begin{array}{c}\text { Before } \\
\text { treatment }\end{array}$ & $\begin{array}{c}\text { After 8-week } \\
\text { treatment }\end{array}$ \\
\hline VDS-total & $48.2 \pm 17.0$ & $26.8 \pm 12.4^{+\dagger}$ & $34.9 \pm 6.2$ & $29.7 \pm 10.1^{+}$ \\
\hline VDS-oral phase & $13.5 \pm 6.3$ & $9.6 \pm 6.5^{+}$ & $8.9 \pm 8.0$ & $6.7 \pm 6.7^{+}$ \\
\hline VDS-pharyngeal phase & $34.5 \pm 12.5$ & $17.2 \pm 9.0^{+\dagger}$ & $28.2 \pm 8.2$ & $26.5 \pm 8.3$ \\
\hline $\begin{array}{l}\text { Anterior displacement } \\
\text { of the hyoid bone }(\mathrm{cm})\end{array}$ & $7.9 \pm 1.7$ & $11.2 \pm 3.0^{* *}$ & $9.0 \pm 4.2$ & $9.4 \pm 4.8$ \\
\hline $\begin{array}{l}\text { Superior displacement } \\
\text { of the hyoid bone }(\mathrm{cm})\end{array}$ & $9.8 \pm 4.3$ & $17.7 \pm 6.9^{* *}$ & $6.8 \pm 2.8$ & $7.9 \pm 3.6$ \\
\hline $\begin{array}{l}\text { Superior displacement } \\
\text { of the larynx }(\mathrm{cm})\end{array}$ & $19.2 \pm 3.5$ & $28.3 \pm 5.6^{* *}$ & $17.1 \pm 3.6$ & $19.1 \pm 4.1$ \\
\hline FOIS & $3.8 \pm 1.5$ & $5.2 \pm 1.1^{+}$ & $4.0 \pm 1.4$ & $4.6 \pm 1.3^{+}$ \\
\hline
\end{tabular}

The values are mean \pm standard deviation. VDS: videofluoroscopic dysphagia scale, FOIS: functional oral intake scale. ${ }^{* *} \mathrm{p}<0.01$ by paired $\mathrm{t}$ test between initial and final scores in the same group. ${ }^{+} \mathrm{p}<0.05,{ }^{+\dagger} \mathrm{p}<0.01$ by Wilcoxon signed-rank test between initial and final scores in the same group.
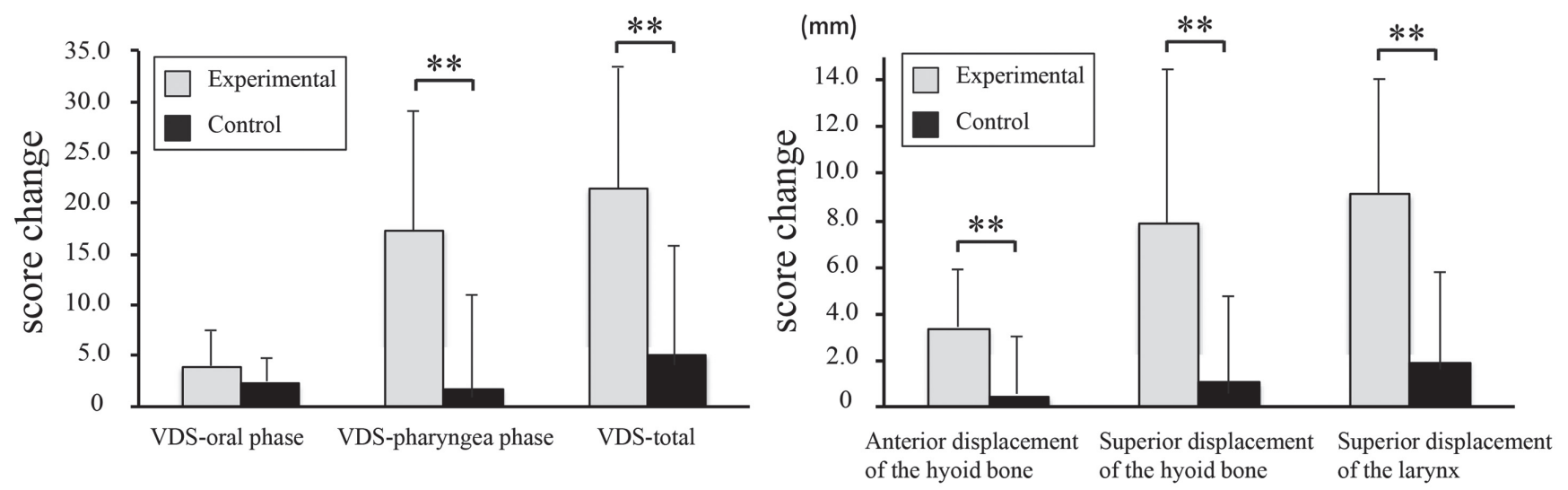

Fig. 4 Comparison of hyoid bone and larynx displacement and videofluoroscopic dysphagia scale (VDS) score changes between the experimental and control groups. Changes for VDS and hyoid bone and larynx displacement following the two interventions are shown. A: VDS, B: hyoid bone and larynx displacement. Graph depicts means \pm standard deviation. Significant differences between the experimental group and the control group are indicated at ${ }^{* *} p<0.01 . p<0.01$ (Mann-Whitney $U$ test between the experimental and control groups).

was also conducted in stroke patients. In contrast to the findings of other groups, they reported no statistically significant differences between traditional dysphagia therapy and VitalStim ${ }^{\circledR}$ (Chattanooga Group, Hixson, Tennessee, USA) therapy. ${ }^{28)}$

Although there has been some controversy in recent studies with respect to its efficacy, elec- trical therapy nonetheless remains widely used as a treatment option for dysphagia. Previous studies have shown that surface electrical stimulation of the laryngeal regions causes significant hyoid and laryngeal descent at rest, and reduces hyoid and laryngeal peak elevation during swallowing. ${ }^{13-15}$ In light of those findings, we chose to develop an 
approach that was different from existing methods of electrical stimulation. In this study, stimulation of the motor points of the thyrohyoid muscle could evoke the inferior displacement of the hyoid bone and superior displacement of the larynx. The thyrohyoid muscle causes downward movement of the hyoid bone, but still elevates the larynx. Our results suggest that we could selectively stimulate the motor points of the thyrohyoid muscle without stimulating the motor point of the sternohyoid muscle. There are several possible explanations for this finding.

First, muscles in the neck are small, short, at greater depth, and are in close proximity to (or superimposed upon) one another. Moreover, the thyrohyoid muscle is thin and is overlaid by the sternohyoid muscle. ${ }^{13)}$ Thus, a surface electrode could be stimulating many different muscles, because the electrical current is likely larger than that required to stimulate any individual superficial muscle in the neck. Therefore, in this study we used a metalsphere probe that could be localized.

Second, HVPC characteristics include twin-peaked paired pulses with high peaks and a low average current. ${ }^{17)}$ The very short pulse duration and high peak of this current can produce an adequate muscle contraction without excitation of pain sensations. ${ }^{17,18)}$ We also considered that a low average current, with enhanced selectivity, could stimulate an anterior neck muscle despite the use of a surface electrode. As reported by Leelamanit et al., the twin-peak pulse also has the particular property of deeper muscle stimulation while avoiding pain stimulation of the neck. ${ }^{16)}$ We considered that the novel NMES system used in this study, which causes less pain and is capable of stimulating deeper muscles, could compensate for the lack of selectivity in surface NMES for treatment of pharyngeal dysphagia.

Moreover, we demonstrated greater improvements in the displacement of the hyoid bone and larynx following NMES combined with conventional treatment than following conventional treatment alone. In this study, we used VFSS to confirm the displacement of the hyoid bone and larynx during electrical stimulation in all subjects. We found that the skin areas above the motor points of the geniohyoid, mylohyoid/anterior belly of the digastric, and thyrohyoid muscles are the most appropriate positions for stimulation to accelerate the anteriorsuperior displacement of the hyoid bone and the larynx. Therefore, we conclude that NMES was able to augment muscular strength.

This study has some limitations that should be considered. The sample size was small, so future studies with a larger number of patients are needed to confirm the results. Also, it is necessary to examine patients with more varied types of diseases. In addition, the study design was a non-randomized controlled design; a randomized controlled study is required to confirm the beneficial effects of this novel NMES system. Moreover, the pulse duration and the frequency were fixed. Because the effects of NMES may be frequency-specific, the effects of varying pulse duration and frequency of electrical stimulation on swallowing physiology require investigation. Further studies will be necessary to confirm the clinical applicability of this novel NMES system as an invasive therapy for dysphagia following brain injury.

\section{Conclusion}

Our novel NMES system, which targeted the skin areas above the motor points of the geniohyoid, mylohyoid/anterior belly of the digastric, and thyrohyoid muscles, caused significant displacement of the hyoid bone and larynx in patients with dysphagia, resulting in improvement of all parameters. The results of this study suggest that NMES combined with conventional treatment is superior to conventional treatment alone for patients with dysphagia after brain injury.

\section{Conflicts of Interest Disclosure}

The authors have no personal, financial, or institutional interest in any of the drugs, materials, or devices in the article.

\section{References}

1) Blumenfeld L, Hahn Y, Lepage A, Leonard R, Belafsky PC: Transcutaneous electrical stimulation versus traditional dysphagia therapy: a nonconcurrent cohort study. Otolaryngol Head Neck Surg 135: 754-757, 2006

2) Carnaby-Mann GD, Crary MA: Adjunctive neuromuscular electrical stimulation for treatment-refractory dysphagia. Ann Otol Rhinol Laryngol 117: 279-287, 2008

3) Freed ML, Freed L, Chatburn RL, Christian M: Electrical stimulation for swallowing disorders caused by stroke. Respir Care 46: 466-474, 2001

4) Kiger M, Brown CS, Watkins L: Dysphagia management: an analysis of patient outcomes using VitalStim therapy compared to traditional swallow therapy. Dysphagia 21: 243-253, 2006

5) Suiter DM, Leder SB, Ruark JL: Effects of neuromuscular electrical stimulation on submental muscle activity. Dysphagia 21: 56-60, 2006

6) Crary MA, Carnaby-Mann GD, Faunce A: Electrical stimulation therapy for dysphagia: descriptive results of two surveys. Dysphagia 22: 165-173, 2007 
7) Humbert IA, Michou E, MacRae PR, Crujido L: Electrical stimulation and swallowing: how much do we know? Semin Speech Lang 33: 203-216, 2012

8) Gallas S, Marie JP, Leroi AM, Verin E: Sensory transcutaneous electrical stimulation improves poststroke dysphagic patients. Dysphagia 25: 291-297, 2010

9) Heijnen BJ, Speyer R, Baijens LW, Bogaardt HC: Neuromuscular electrical stimulation versus traditional therapy in patients with Parkinson's disease and oropharyngeal dysphagia: effects on quality of life. Dysphagia 27: 336-345, 2012

10) Lin PH, Hsiao TY, Chang YC, Ting LL, Chen WS, Chen SC, Wang TG: Effects of functional electrical stimulation on dysphagia caused by radiation therapy in patients with nasopharyngeal carcinoma. Support Care Cancer 19: 91-99, 2011

11) Park JW, Kim Y, Oh JC, Lee HJ: Effortful swallowing training combined with electrical stimulation in post-stroke dysphagia: a randomized controlled study. Dysphagia 27: 521-527, 2012

12) Ryu JS, Kang JY, Park JY, Nam SY, Choi SH, Roh JL, Kim SY, Choi KH: The effect of electrical stimulation therapy on dysphagia following treatment for head and neck cancer. Oral Oncol 45: 665-668, 2009

13) Kagaya H, Baba M, Saitoh E, Okada S, Yokoyama M, Muraoka Y: Hyoid bone and larynx movements during electrical stimulation of motor points in laryngeal elevation muscles: a preliminary study. Neuromodulation 14: 278-283; discussion 283, 2011

14) Humbert IA, Poletto CJ, Saxon KG, Kearney PR, Crujido L, Wright-Harp W, Payne J, Jeffries N, Sonies BC, Ludlow CL: The effect of surface electrical stimulation on hyolaryngeal movement in normal individuals at rest and during swallowing. J Appl Physiol 101: 1657-1663, 2006

15) Ludlow CL, Humbert I, Saxon K, Poletto C, Sonies B, Crujido L: Effects of surface electrical stimulation both at rest and during swallowing in chronic pharyngeal Dysphagia. Dysphagia 22: 1-10, 2007

16) Leelamanit V, Limsakul C, Geater A: Synchronized electrical stimulation in treating pharyngeal dysphagia. Laryngoscope 112: 2204-2210, 2002

17) Alon G: High voltage stimulation. Effects of electrode size on basic excitatory responses. Phys Ther 65: 890-895, 1985

18) Wong RA: High voltage versus low voltage electrical stimulation. Force of induced muscle contraction and perceived discomfort in healthy subjects.
Phys Ther 66: 1209-1214, 1986

19) Gilcreast DM, Stotts NA, Froelicher ES, Baker LL, Moss KM: Effect of electrical stimulation on foot skin perfusion in persons with or at risk for diabetic foot ulcers. Wound Repair Regen 6: 434-441, 1998

20) Gomes NC, Berni-Schwarzenbeck KC, Packer AC, Rdrigues-Bigaton D: Effect of cathodal high-voltage electrical stimulation on pain in women with TMD. Rev Bras Fisioter 16: 10-15, 2012

21) Kloth LC, Feedar JA: Acceleration of wound healing with high voltage, monophasic, pulsed current. Phys Ther 68: 503-508, 1988

22) Toyama K, Matsumoto S, Kurasawa M, Noma T, Shimodozono M, Kawahira K: [Effect of neuromuscular electrical stimulation on hyoid bone and laryngeal movements to improve swallowing function]. Sogo Rihabiriteshon 39: 977-985, 2011 (Japanese)

23) Robbins J, Kays SA, Gangnon RE, Hind JA, Hewitt AL, Gentry LR, Taylor AJ: The effects of lingual exercise in stroke patients with dysphagia. Arch Phys Med Rehabil 88: 150-158, 2007

24) Han TR, Paik NJ, Park JW, Kwon BS: The prediction of persistent dysphagia beyond six months after stroke. Dysphagia 23: 59-64, 2008

25) Crary MA, Mann GD, Groher ME: Initial psychometric assessment of a functional oral intake scale for dysphagia in stroke patients. Arch Phys Med Rehabil 86: 1516-1520, 2005

26) Lim KB, Lee HJ, Lim SS, Choi YI: Neuromuscular electrical and thermal-tactile stimulation for dysphagia caused by stroke: a randomized controlled trial. J Rehabil Med 41: 174-178, 2009

27) Shaw GY, Sechtem PR, Searl J, Keller K, Rawi TA, Dowdy E: Transcutaneous neuromuscular electrical stimulation (VitalStim) curative therapy for severe dysphagia: myth or reality? Ann Otol Rhinol Laryngo 1116: 36-44, 2007

28) Bülow M, Speyer R, Baijens L, Woisard V, Ekberg O: Neuromuscular electrical stimulation (NMES) in stroke patients with oral and pharyngeal dysfunction. Dysphagia 23: 302-309, 2008

Address reprint requests to: Shuji Matsumoto, MD, PhD, Kirishima Rehabilitation Center of Kagoshima University Hospital, 3930-7 Takachiho, Makizonocho, Kirishima, Kagoshima 899-6603, Japan. e-mail: shushu@m.kufm.kagoshima-u.ac.jp 